\title{
Rare Pancreatic Tumors
}

\author{
Amitkumar Choudhari ${ }^{1,2}$ Pooja Kembhavi ${ }^{1,2}$ Mukta Ramadwar ${ }^{3,4}$ Aparna Katdare ${ }^{1,2}$ \\ Vasundhara Smriti ${ }^{1,2}$ Akshay D. Baheti ${ }^{1,2}$
}

1Department of Radiodiagnosis, Tata Memorial Hospital, Mumbai,

Maharashtra, India

2Department of Radiodiagnosis, Homi Bhabha National University,

Mumbai, Maharashtra, India

${ }^{3}$ Department of Pathology, Tata Memorial Hospital, Mumbai,

Maharashtra, India

${ }^{4}$ Department of Pathology, Homi Bhabha National University,

Mumbai, Maharashtra, India

Address for correspondence Akshay D. Baheti, MD, Department of Radiodiagnosis, Tata Memorial Hospital, Ernest, Borges Marg Parel Mumbai 400012, India (e-mail: akshaybaheti@gmail.com).

J Gastrointestinal Abdominal Radiol ISGAR 2020;3:64-74
Abstract
Keywords
- pancreatic cancer
- uncommon
- pancreatoblastoma
- acinar cell
- lymphoma

Pancreatic ductal adenocarcinoma, neuroendocrine tumor, and cystic pancreatic neoplasms are the common pancreatic tumors most radiologists are familiar with. In this article we review the clinical presentation, pathophysiology, and radiology of rare pancreatic neoplasms. While the imaging features are usually nonspecific and diagnosis is based on pathology, the radiology along with patient demographics, history, and laboratory parameters can often help indicate the diagnosis of an uncommon pancreatic neoplasm and guide appropriate management in these cases.

\section{Introduction}

Pancreatic tumors of various histological subtypes can be encountered in clinical practice, most common being pancreatic ductal adenocarcinoma (PDAC), which constitutes $85 \%$ of all pancreatic neoplasms. ${ }^{1}$ Histologically pancreatic neoplasms can be of epithelial and nonepithelial origin (-Table 1). The less common epithelial neoplasms will be discussed in this review, include acinar cell neoplasm, adenosquamous carcinoma, and pancreatoblastoma. Nonepithelial neoplasms can arise from intrapancreatic or peripancreatic mesenchymal, hematopoietic, or neural elements. These account for only 1 to $2 \%$ of all pancreatic neoplasms, and will also be discussed. ${ }^{2-4}$

\section{Epithelial Neoplasms}

\section{Pancreatic Acinar Cell Tumor}

Acinar cell carcinoma (ACC) comprises only $1 \%$ of all pancreatic neoplasms. The neoplasm is usually encountered in the fifth to seventh decade of life, with a stark male preponderance. It is a neoplasm of the exocrine pancreas, but can occasionally also have an endocrine component. The tumor cells can secrete pancreatic enzymes, especially lipase. Resulting hyperlipasemia may rarely lead to extraabdominal manifestations like ectopic subcutaneous fat necrosis and polyarthritis (lipase hypersecretion syndrome). ${ }^{4}$

These tumors are hypoenhancing compared with the pancreas and are frequently associated with cystic or necrotic areas as well as calcifications $\mathbf{s}^{5,6}$ ( $\mathbf{- F i g . ~ 1 ) . ~ T h e y ~ a r e ~ u s u a l l y ~}$ well-circumscribed, a feature that can be useful in differentiating ACC from PDAC, with the latter being poorly defined and infiltrative. ${ }^{5-7}$ No specific anatomic location of predilection in the pancreas has been identified. ${ }^{6}$ The tumors are usually large at presentation, with a mean of $6 \mathrm{~cm} .{ }^{7}$ Ductal obstruction is uncommon, despite their large size, ${ }^{8}$ also unlike PDAC. Due to their lower propensity for vascular, lymph node, and adjacent soft tissue invasion, gross total resection can more often be achieved. ${ }^{9}$

\section{Adenosquamous Carcinoma of the Pancreas}

Adenosquamous carcinoma of the pancreas is another rare neoplasm of the pancreas, constituting 1 to $4 \%$ of adenocarcinomas. ${ }^{10}$ It has a slight male preponderance and is found in the seventh decade of life. The diagnosis is based on the presence of $\geq 30 \%$ squamous component within the lesion on pathology. ${ }^{11}$ Signs and symptoms like weight loss, anorexia, jaundice, and abdominal and back pain are similar to PDAC.
DOI https://doi.org/ 10.1055/s-0040-1708577 ISSN 2581-9933.
License terms

()(1) $\Theta \circledast$ 
Table 1 World Health Organization classification of pancreatic tumors

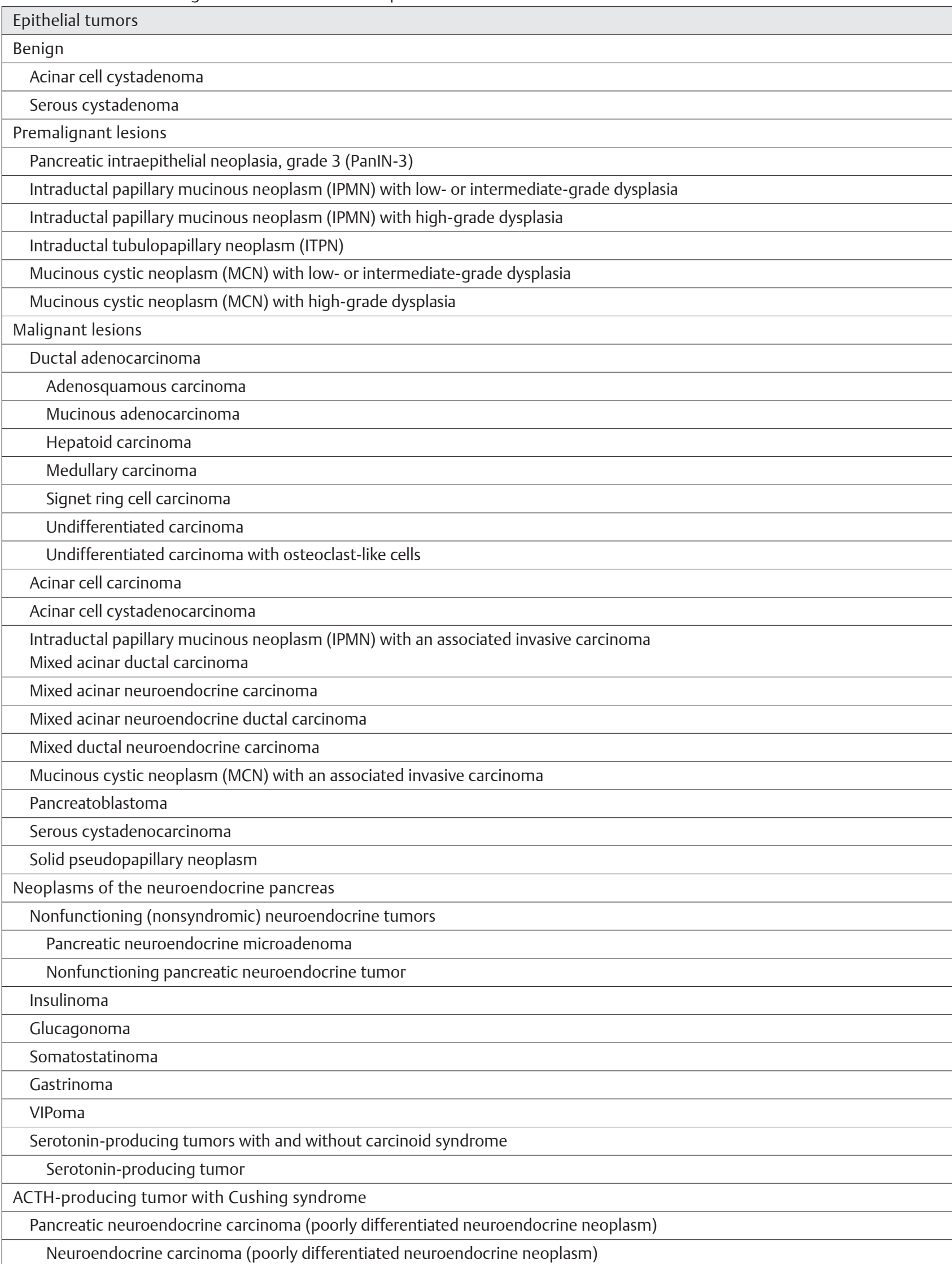


Table 1 (continued)

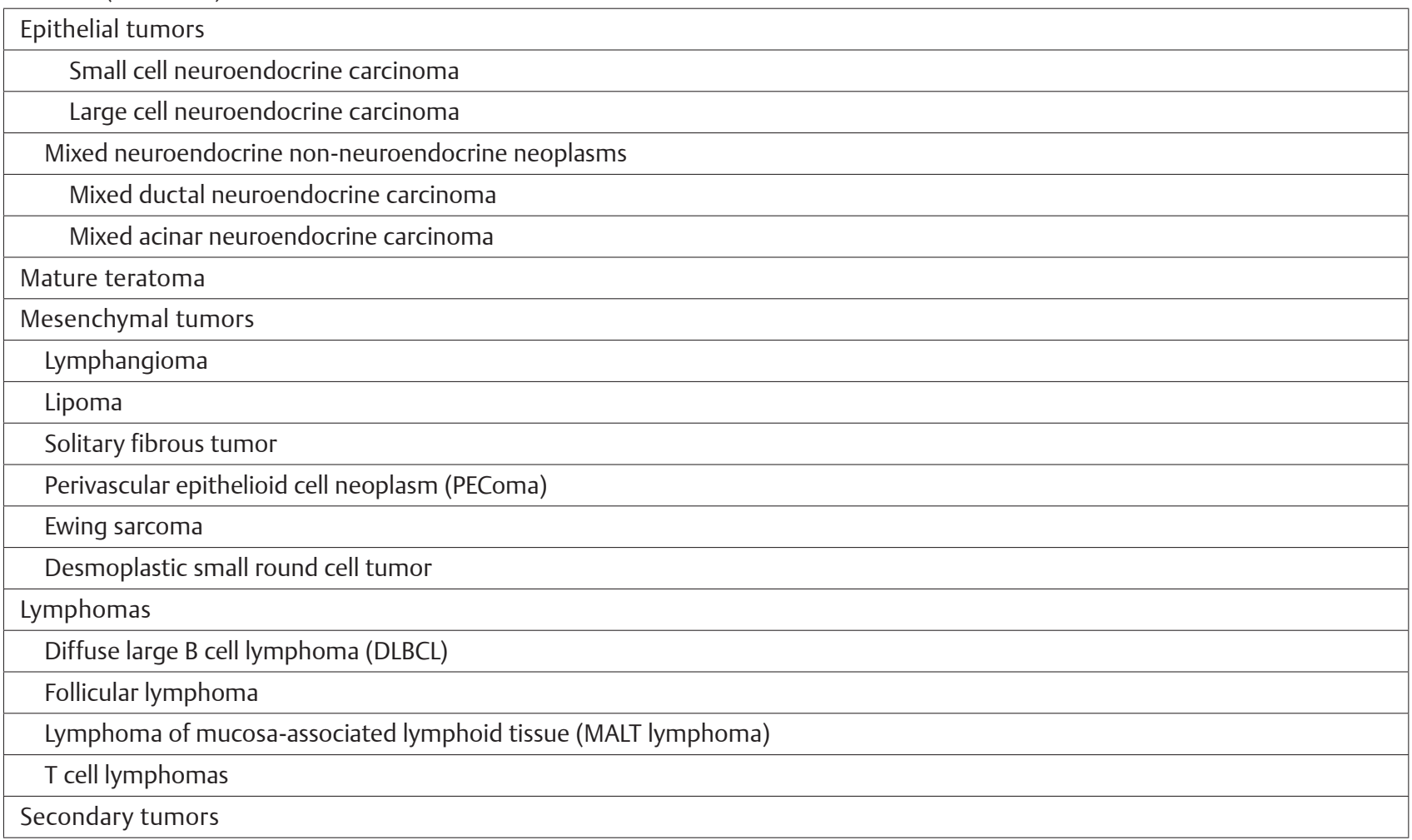
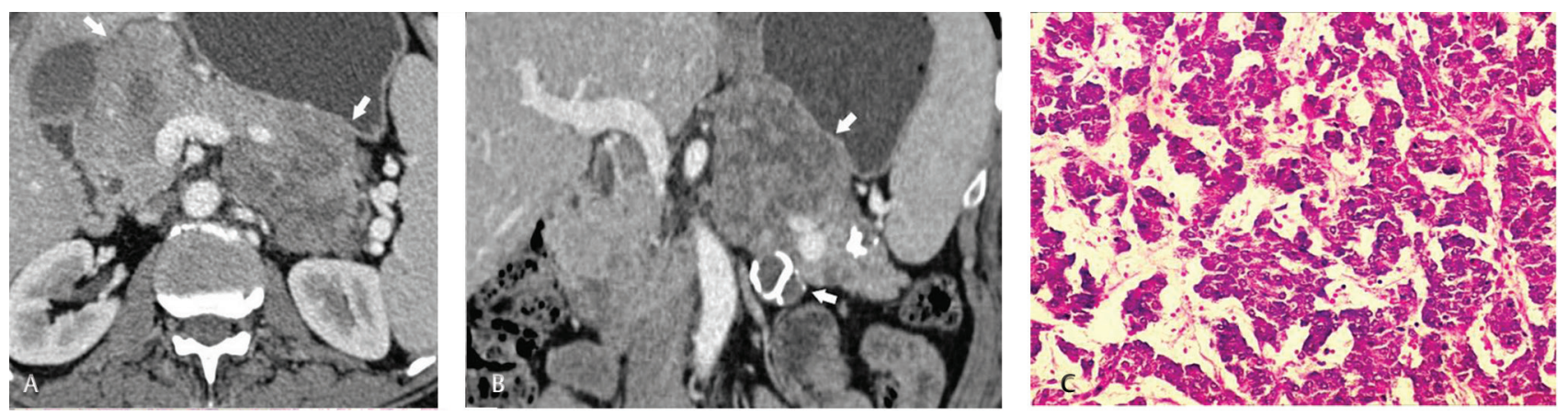

Fig. 1 (A, B) A 45-year-old man with pain in the abdomen. Axial and coronal contrast-enhanced computed tomography (CECT) abdomen images reveal two heterogeneously enhancing masses arising from the head and the body of the pancreas with calcifications within (arrows). Biopsy of the lesions confirmed the diagnosis of acinar cell carcinoma. (C) Hematoxylin and eosin stained section (20x) shows epithelial cells arranged in an acinar pattern. Cells show eosinophilic cytoplasm, small round nuclei with low mitotic activity, consistent with acinar cell carcinoma.

However, the prognosis is poorer compared with PDAC. ${ }^{10}$ On imaging, adenosquamous carcinomas are round-lobulated lesions associated with extensive necrosis. Tumor thrombosis of portal venous (PV) system can help to differentiate these lesions from PDAC; the latter tends to encase the vessels and cause occlusion of PV system due to bland thrombosis. ${ }^{11}$ The differential diagnosis also includes anaplastic/undifferentiated carcinoma, neuroendocrine carcinoma, and acinar cell carcinoma, among others.

\section{Pancreatoblastoma}

Pancreatoblastoma is a rare primary neoplasm of the pancreas occurring in childhood. The tumor is usually seen in 1 to 8 years age group. Pancreatoblastoma arises from acini which resemble the tissue of fetal pancreas at 7 weeks gestation. Clinically, it presents as a large abdominal mass with pressure symptoms like pain, early satiety, obstructive jaundice, vomiting, and constipation. The tumor usually does not cause bowel or biliary obstruction despite its large size, since it has a soft, gelatinous consistency. The $\alpha$ fetoprotein levels can be elevated in 25 to $55 \%$ cases. Congenital pancreatoblastoma can be associated with Beckwith-Wiedemann syndrome, where the tumor can be cystic. ${ }^{12}$ Anatomically, the lesion can occur in any part of the pancreas or involve the entire organ.

Ultrasound usually demonstrates a heterogeneous complex cystic/necrotic mass with thick echogenic septa. On computed tomography (CT)/magnetic resonance imaging (MRI), the lesion presents as a well-defined or a partially circumscribed, lobulated mass with enhancing septa on 
$\mathrm{CT}^{8,13}$ ( - Fig. 2). There is a propensity for adjacent soft tissue invasion, including involvement of the duodenum, adrenals, spleen, and even neurovascular infiltration. Calcifications can also be seen, and are usually rim-like or clustered if present. ${ }^{12}$ Metastases to liver, omentum, and peritoneum can occur. In large masses, it can often be difficult to ascertain whether the pancreas is the organ of origin or just secondarily involved. Differentials like neuroblastoma, Wilms tumor, or non-Hodgkin's lymphoma (NHL) would need to be considered, and a biopsy may be necessary. Neuroblastoma can be differentiated MIBG positivity on nuclear imaging study, presence of urine catecholamines, and paraneoplastic syndromes like opsoclonus-myoclonus. Wilms tumor may be differentiated by its propensity for venous invasion, pulmonary metastasis, and renal origin. NHL can also present as a large retroperitoneal mass which may involve the pancreas. Lack of necrosis and calcification within the mass can help to differentiate NHL from other retroperitoneal masses in children. Surgery is the treatment of choice. ${ }^{12,13}$

\section{Hematolymphoid Tumors}

Pancreatic involvement by hematolymphoid malignancies like lymphomas, leukemias, and plasmacytomas are rare but can occur.

\section{Pancreatic Lymphoma}

Pancreatic lymphoma can arise primarily in the pancreas or secondarily involve pancreas in the presence of systemic disease (-Figs. 3 and 4). Primary pancreatic lymphoma is an exceedingly rare neoplasm, representing only $0.1 \%$ of malignant lymphomas and $0.2 \%$ of primary pancreatic tumors. ${ }^{14}$ The Dawson criteria ${ }^{15}$ for diagnosis of primary pancreatic lymphoma include lymphoma arising as a mass within the pancreas and peripancreatic lymph nodes with no liver or spleen involvement, no superficial or mediastinal lymphadenopathy, and a normal leukocyte count. Patients can be asymptomatic or present with abdominal pain. B symptoms like fever, night sweats, and weight loss can also be encountered. Occasionally patients can also present with gastric outlet obstruction, or rarely, GI bleeding. ${ }^{16}$
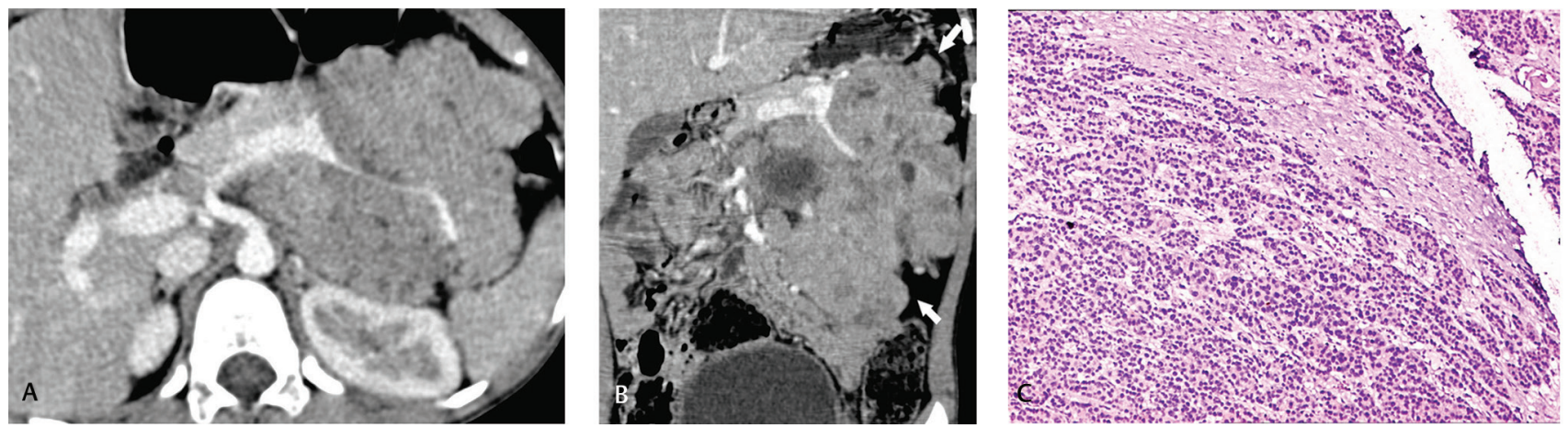

Fig. 2 (A, B) A 9-year-old boy presenting with an incidentally detected abdominal lump. Axial and coronal (CECT) images show a large heterogeneous predominantly solid mass arising from the tail of the pancreas and encasing and narrowing the splenic vein (arrows). It demonstrates a cystic/necrotic focus within (B). The possibility of a pancreatoblastoma was raised given the patient's age and the imaging features. The patient underwent distal pancreaticosplenectomy, which confirmed the diagnosis. (C) Hematoxylin and eosin-stained section (10×) shows a cellular tumor composed of uniform tumor cells arranged in acini and cords. Nuclear pleomorphism and mitotic activity are not conspicuous. Findings are diagnostic of pancreatoblastoma.
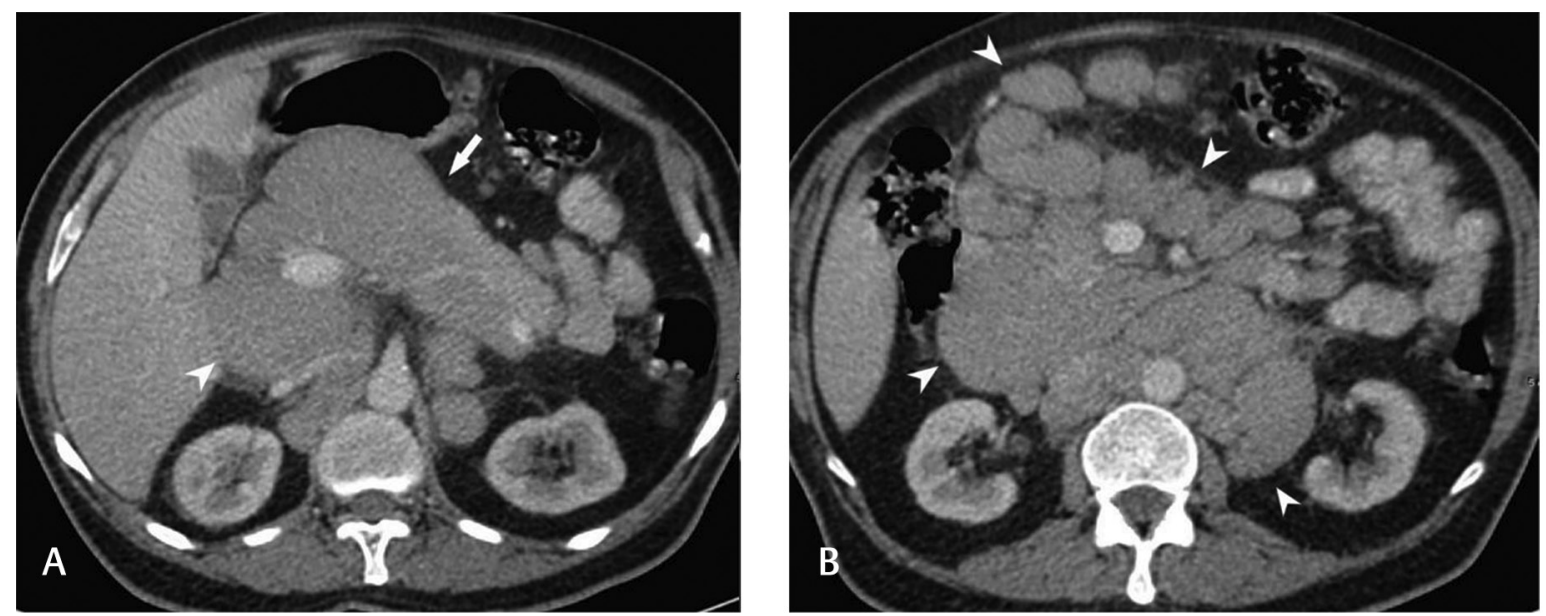

Fig. 3 A 54-year-old man with abdominal pain and weight loss. Axial CECT images revealed a large infiltrative homogeneously enhancing lesion (arrow) in the body of the pancreas (A). There were multiple discrete homogeneous enlarged retroperitoneal and mesenteric nodes (arrowheads) present as well (B), consistent with the diagnosis of lymphoma with secondary pancreatic involvement. Biopsy revealed chronic lymphocytic lymphoma. 


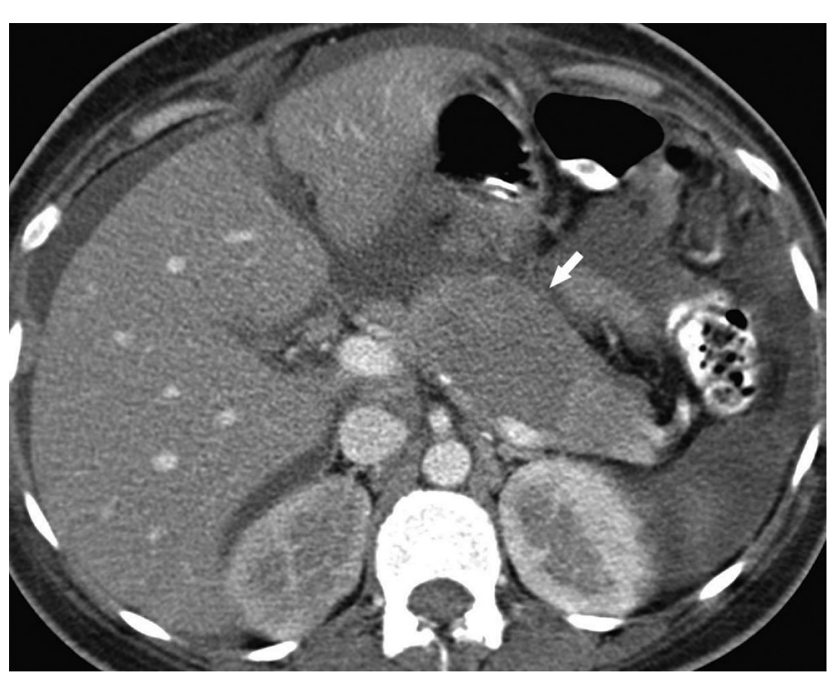

Fig. 4 A 37-year-old woman with abdominal pain. Axial CECT abdomen image reveals a well-defined hypoenhancing lesion (arrow) in the body of the pancreas without ductal dilatation or calcification. There is mild ascites present as well. Biopsy revealed diffuse large B cell lymphoma.

Pathologically, the diffuse large B cell lymphoma is more common. Other less common variants which may be encountered include follicular and marginal zone lymphoma. ${ }^{14}$ Diagnostic confirmation through percutaneous/endoscopic US-guided biopsy or surgical biopsy is important, because pancreatic lymphoma needs to be treated by chemotherapy, whereas pancreatic adenocarcinoma treatment warrants surgery with or without chemoradiation. ${ }^{17}$

Although PPL can arise anywhere in the pancreas, the incidence $s$ higher in the head of pancreas due to abundance of lymphoid tissue in the head. PPL is usually large at presentation. On ultrasonography (USG), pancreatic lymphoma appears as bulky homogeneous hypoechoic mass confined to the pancreas, without posterior acoustic enhancement. On cross-sectional imaging, PPL can be present as a focal well-defined soft tissue mass or a diffuse, ill-defined, infiltrative mass. CT will demonstrate a homogeneously hypoenhancing mass, without any calcification or necrosis in most cases ( - Fig. 4). Ductal dilatation and vascular occlusion/invasion are absent just like lymphomas elsewhere in the body, despite the large tumor size. This finding is in stark contrast to imaging features of adenocarcinoma, which cause ductal dilatation even when much smaller in size. MRI offers no additional advantage over $\mathrm{CT}$ in characterizing the lesion..$^{16}$ On FDG PET/CT, (fluorodeoxy glucose - positron emission tomgraphy) pancreatic lymphoma is an avid tumor with various patterns of FDG uptake-focal nodular uptake (solitary or multifocal), segmental uptake, or diffuse uptake. PDAC can also demonstrate focal FDG uptake; however, lymphoma tends to be much more FDG avid with maximal standardized uptake values $\left(\mathrm{SUV}_{\max }\right.$ ) reportedly ranging from 7.4 to 26.5 , compared with pancreatic adenocarcinoma, which is relatively less FDG avid with SUV $_{\text {max }}$ ranging from 2 to $12 .{ }^{18}$ There remains an overlap though, and biopsy is needed for diagnosis.

The main differential for PPL is PDAC, given its higher incidence. Imaging features like large size, homogeneous appearance, lack of ductal dilatation, absence of invasion/ occlusion of the portal, superior mesenteric, or splenic vein despite encasement indicates PPL over PDAC. Infrarenal adenopathy, below the level of renal veins, is also suggestive of pancreatic lymphoma. Presence of a mass, which infiltrates intra-abdominal organs, crosses anatomic boundaries, and stretches vessels without encasement/thrombosis, is also more likely to represent pancreatic lymphoma. Autoimmune pancreatitis is another differential for smaller lesions. ${ }^{16}$

\section{Plasmacytoma}

Pancreas is a rare site of extramedullary or solitary plasmacytoma, which occurs due to monoclonal proliferation of plasmocytes outside the marrow. ${ }^{19}$ Pancreatic involvement by plasmacytoma/multiple myeloma is exceedingly rare, accounting for less than $0.1 \%$ of pancreatic masses.

Pancreatic plasmacytoma tends to affect middle-aged men (median age, 55 years). These can be solitary, where head is the usual site, or multifocal. Plasmacytomas frequently secrete M-protein, which is detectable in serum or urine. Patients with pancreatic head plasmacytoma can present with obstructive jaundice, similar to PDAC. Plasmacytomas demonstrate immunoreactivity to CD38 and CD138 and $\kappa$ or $\lambda$ light chain restriction. ${ }^{2}$ On USG, the lesion is seen as hypoechoic mass associated with biliary dilatation. Plasmacytomas are usually hypo- and isoenhancing masses on CT and MRI but may demonstrate arterial phase hyperenhancement ( - Fig. 5). They are hypointense on T2-weighted images
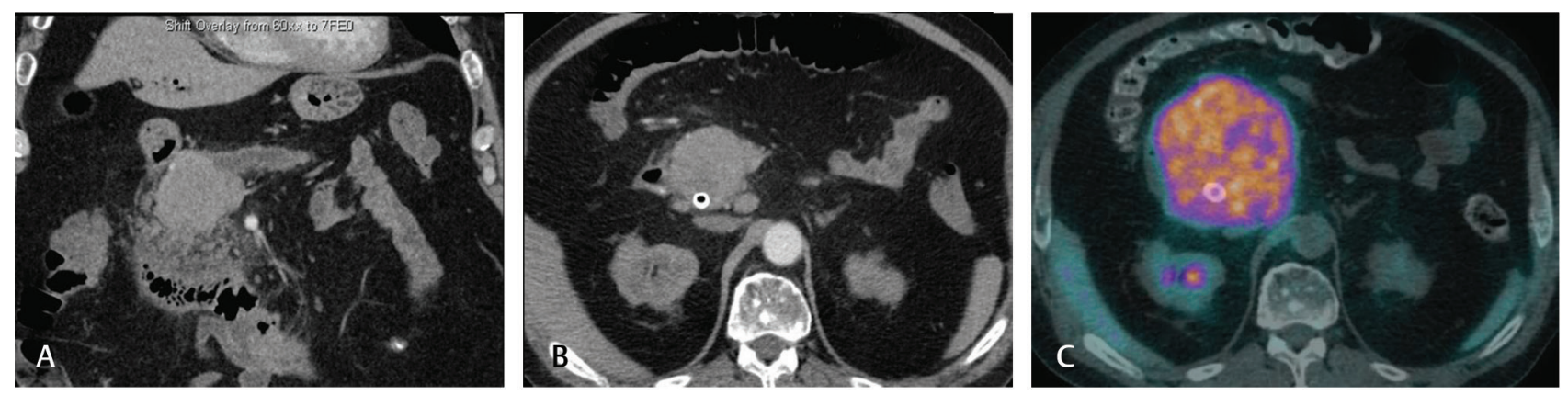

Fig. 5 (A-C) A 54-year-old man with vague abdominal pain and weight loss. Axial and coronal CECT images (A, B) reveals a well-defined homogeneous isohyperattenuating pancreatic head mass, with moderate upstream pancreatic ductal dilatation. There was no calcification. Biopsy revealed plasmacytoma. Subsequent FDG PET/CT performed demonstrates an intensely FDG-avid mass (C), which has increased in size in the interim. (Image courtesy Dr. Atul Shinagare). 
due to their high cellularity and will show diffusion restriction, a clue to the diagnosis. ${ }^{19}$ They demonstrate intense FDG avidity on PET/CT ( Fig. 5C). ${ }^{19}$

The imaging differentials for a plasmacytoma would include primary pancreatic lymphoma, primitive neuroendocrine tumor, sarcoma, and metastasis. Biopsy remains essential for diagnosis.

\section{Mesenchymal Tumors}

Mesenchymal tumors, including both benign/less aggressive tumors and sarcomas, uncommonly involve the pancreas, and usually present as indeterminate heterogeneous masses which can be differentiated only on histopathology. We describe a few pancreatic mesenchymal tumors.

\section{Perivascular Epithelioid Cell Tumor}

Perivascular epithelioid cell tumors (PEComas) comprise a rare family of mesenchymal tumors found at various anatomic sites, sharing the distinctive perivascular epithelioid cells, which demonstrate characteristic immunophenotype and morphology. They are also known as "sugar tumors" due to the glycogen rich cytoplasm of the perivascular epithelioid cells. ${ }^{20,21}$ PEComas are frequently associated with germline mutations in the tuberous sclerosis complex genes. ${ }^{22}$ The neoplasm has a strong female preponderance. On imaging, PEComas present as large well-defined, encapsulated, and hypoenhancing masses. ${ }^{20}$ There are no characteristic imaging features to help differentiate them from other neoplasms.

\section{Solitary Fibrous Tumor}

Solitary fibrous tumors (SFTs) are rare mesenchymal tumors, constituting less than $1 \%$ of pancreatic neoplasms. Initially, thought to be an exclusively pleural neoplasm, it is now established that it can arise in any anatomical site in the body, extrapleural SFTs being more common. ${ }^{23}$ On pathology, SFTs are composed of whorled pattern of spindle cells and collagen, which comprises the fibrous variant, whereas the cellular form has a patternless or monotonous appearance. The fibrous form exhibits strong CD34 immunoreactivity compared with the cellular variant and is $\mathrm{S} 100$ negative. Malignant SFTs, however, lose CD34 immunoreactivity and overexpress S100, in addition to demonstrating cellular atypia, high mitotic activity, and higher frequency of necrotic and hemorrhagic areas.

Pancreatic SFTs, similar to other extrathoracic SFTs have a female predilection. Usually, these tumors can be encountered in the 41 to 78 years of age group, varying in size from 2 to $8 \mathrm{~cm}$, but are generally large at presentation. ${ }^{23}$ They are slow-growing tumors, and the patients can present with abdominal distension, bowel obstruction, or constipation once the tumor becomes large in size. Obstructive jaundice is uncommon. Less than $5 \%$ of patients with SFTs, especially malignant tumor, may present with hypoglycemia due to secretion of insulin-like growth factor (Doege-Potter syndrome). Other symptoms can include clubbing, hypertrophic osteoarthropathy, and arthralgia. ${ }^{24}$

On imaging, pancreatic SFTs are seen as well-circumscribed hypervascular masses, with the cellular tumors demonstrating prominent arterial phase enhancement and both subtypes showing persistent progressive enhancement on the venous and delayed phases. Mixed pattern of enhancement with nonenhancing cystic/necrotic changes can be seen as well in large tumors. On catheter angiography, the lesion is hypervascular with arborizing vessels arising from a vascular stalk, along with dilated feeding arteries and early draining veins. Hence preoperative embolization may be useful in reducing intraoperative blood loss. ${ }^{23}$ Imaging differentials include nonfunctioning pancreatic neuroendocrine tumors, solid pseudopapillary neoplasm, and occasionally hypervascular metastasis; biopsy is needed for a definitive diagnosis. Surgery is the treatment of choice.

\section{Neurogenic Tumors}

\section{Neurofibroma}

Neurofibromas are neurogenic tumors composed of all components of the peripheral nerve, including Schwann cells, fibroblasts, perineural cells, and axons. ${ }^{3}$ Neurofibromas are associated with neurofibromatosis type 1 (von Recklinghausen disease), an autosomal dominant disorder predisposing to formation of plexiform neurofibromas. Pancreatic involvement can rarely occur by intraparenchymal growth of peripheral neurofibroma, which can result in abdominal pain and duct obstruction..$^{25}$

Neurofibromas are homogeneously hypoattenuating and show mild contrast enhancement on CT scan. On MRI, neurofibromas are hypointense on $\mathrm{T} 1$ and heterogeneously hyperintense on T2-weighted images. Target appearance may be appreciated on T2-weighted images as central hypointensity with high peripheral T2 signal, corresponding to central well-organized tissue. Whorled appearance with T2 hypointense curvilinear areas may be seen, corresponding to bundles of collagen and Schwann cells, may also be encountered. Malignant transformation is rare and can be suggested by include rapid growth, necrosis, hemorrhage, calcification, loss of the "target sign" (due to loss of cellular organization), and invasion into adjacent structures with associated edema. Surgical excision, though challenging, is the standard treatment.

\section{Schwannoma}

Schwannomas are benign nerve sheath tumors, entirely composed of the myelin-producing neurilemma cells. ${ }^{26}$ Usually solitary, multiple schwannomas can be associated with type 2 neurofibromatosis. Schwannomas can be found at almost every location of the body including in extremities, head, neck, retroperitoneum, mediastinum, pelvis, and rectum. Visceral involvement is rare. These neoplasms are usually found in adults, without any gender predilection. Due to their slow growing nature, pancreatic Schwannomas are usually large at presentation with an average size of $9 \mathrm{~cm}$ (range: $2-20 \mathrm{~cm}$ ). ${ }^{3}$ Clinically, patients can present with vague abdominal pain, although anorexia, back pain, vomiting, weight loss, and gastrointestinal bleeding. ${ }^{27} \mathrm{His}-$ tologically, schwannomas have two main features: highly cellular "Antoni A" areas and a sparsely cellular, myxoid, or 
"Antoni B" component. Schwannomas exhibit strong S-100 immunoreactivity. 3,27

On CT and MRI, pancreatic schwannomas reflect their architecture-the cellular Antoni A schwannomas are solid lesions with heterogeneous enhancement, whereas the Antoni B-predominant schwannomas are homogeneous cystic or multiseptated masses. ${ }^{3}$ Two-thirds of pancreatic schwannomas undergo degenerative changes such as cyst formation, necrosis, calcification, and hemorrhage, and these changes can mimic pancreatic cystic tumors including cystic neuroendocrine tumors, ${ }^{26}$ needing histopathological confirmation. At MRI, pancreatic schwannomas are T1 hypointense and T2 hyperintense. On contrast-enhanced study, Antoni A schwannomas reveal intense contrast enhancement, whereas Antoni B schwannomas show little enhancement, with nonenhancing, T2-hyperintense cystic areas. ${ }^{3,28}$

Conservative surgery like enucleation, where achievable, can be curative, due to benign nature of the neoplasm. A more radical approach may be warranted when rapid growth, infiltration of adjacent structures, and prominent necrotic and hemorrhagic changes within the tumor are encountered, suggesting malignant transformation. ${ }^{26}$

\section{Hemangioma}

Pancreatic hemangiomas are exceedingly rare neoplasms, which may be encountered in the pediatric age group and to a lesser extent in adults. Usually detected in infancy, the lesion can evolve through a proliferative phase in infancy to an involuting phase of variable duration lasting up to 12 years of age, finally reducing to a fibrofatty residuum in adulthood. ${ }^{29}$ Multiphase contrast-enhanced CT scan/MRI can reveal typical irregular enhancement with centripetal fill-in in the infantile hemangioma. Adult hemangiomas, however, do not reveal arterial enhancement, unlike hepatic hemangioma, with or without delayed enhancement suggesting a cystic neoplasm. Multiphase CT cannot exclude pancreatic hemangioma. ${ }^{30,31}$ Low T1 signal and high T2 signal may be considered suggestive of hemangioma in an enhancing lesion..$^{30}$ Diagnosis is usually confirmed at histopathology, with the lesion expressing CD31 and CD34 immunoreactivity. ${ }^{31}$ A more conservative surgical approach can be considered in such neoplasm instead of extensive procedure like Whipple's surgery in patients who are clinically symptomatic or present with complications like hemorrhage, mass effect like obstructive jaundice.

\section{Mesenchymal Sarcomas}

Sarcomas of the pancreas are exceedingly rare, amounting to $0.1 \%$ of pancreatic neoplasms. ${ }^{2}$ Except for primitive neuroectodermal tumors and rhabdomyosarcomas, which are encountered in children and young adults, most pancreatic sarcomas predominantly affect the elderly, with a median age of 65 years at diagnosis. ${ }^{2}$ Clinically, most patients present with vague abdominal pain and a large indeterminate mass. The mass is usually large at presentation, and to differentiate a primary retroperitoneal mass secondarily involving the pancreas from a primary pancreatic mass. A mass epicentered in the pancreas is assumed to be pancreatic/peripancreatic in origin. We discuss some "common" sarcomas. Histopathology remains essential for diagnosis.

\section{Leiomyosarcoma}

Leiomyosarcoma is the most common type of pancreatic sarcoma, thought to arise from the smooth muscles of the pancreatic duct or small intrapancreatic blood vessels. Pancreas can also be secondarily involved by leiomyosarcoma originating in the retroperitoneum, mimicking a primary pancreatic neoplasm.

On imaging, these tumors are usually large and heterogeneous, with peripheral hypervascularity and central areas of cystic degeneration/necrosis (-Fig. 6). On MRI, the lesions are hypointense to the pancreas on $\mathrm{T} 1$-weighted images and hyperintense on T2-weighted images. Usually, these tumors present with distant metastasis, which can be detected with high sensitivity using ${ }^{18} \mathrm{~F}-\mathrm{FDG}$ PET/CT. ${ }^{2}$

\section{Extraskeletal Ewing's Sarcoma/Primitive Neuroectodermal Tumor}

Extraskeletal Ewing's sarcoma (EES) and primitive neuroectodermal tumors are rare aggressive tumors arising from ectopic neural and neuroectodermal tissue and can occur anywhere in the body. They occur almost exclusively in children and young adults. ${ }^{3}$ Because of their similar morphology, immunohistochemistry, and cytogenetic alterations $(t[11 ; 22])$, these tumors are documented together with the Ewing's sarcoma family.

These neoplasms present as large solid masses with cystic degeneration and necrosis, similar to other sarcomas. Peripheral hyperenhancement may be seen. ${ }^{32}$ They are FDG avid on PET/CT, a useful modality for detection of the primary tumor, postresection recurrence, and metastases. ${ }^{3}$

\section{Undifferentiated Pleomorphic Sarcoma}

Undifferentiated pleomorphic sarcoma is a common retroperitoneal sarcoma, more common in the elderly, and can rarely arise from the pancreas. On imaging, the tumor may present as large, heterogeneous mass with necrotic components. Calcification may be present. ${ }^{3}$ The tumor is FDG avid on PET/CT. It will remain an indeterminate pancreatic malignancy on imaging, and histopathology is necessary for diagnosis.

\section{Metastasis}

Pancreatic metastases account for only 2\% of pancreatic neoplasms. Most common tumors metastasizing to the pancreas include renal, lung, breast, and melanoma. ${ }^{33}$ Renal cell carcinoma and melanoma metastases are often hypervascular. It is unlikely for pancreatic metastasis to present before the detection of primary neoplasm. Metastases, particularly renal cell carcinoma metastases, can occur even several years following the resection of the primary, with recurrences being reported even 12 to 32 years after surgery ( - Fig. $\mathbf{7}){ }^{33}$

Patients are often asymptomatic, with the pancreatic metastases being an "incidental" finding on surveillance 

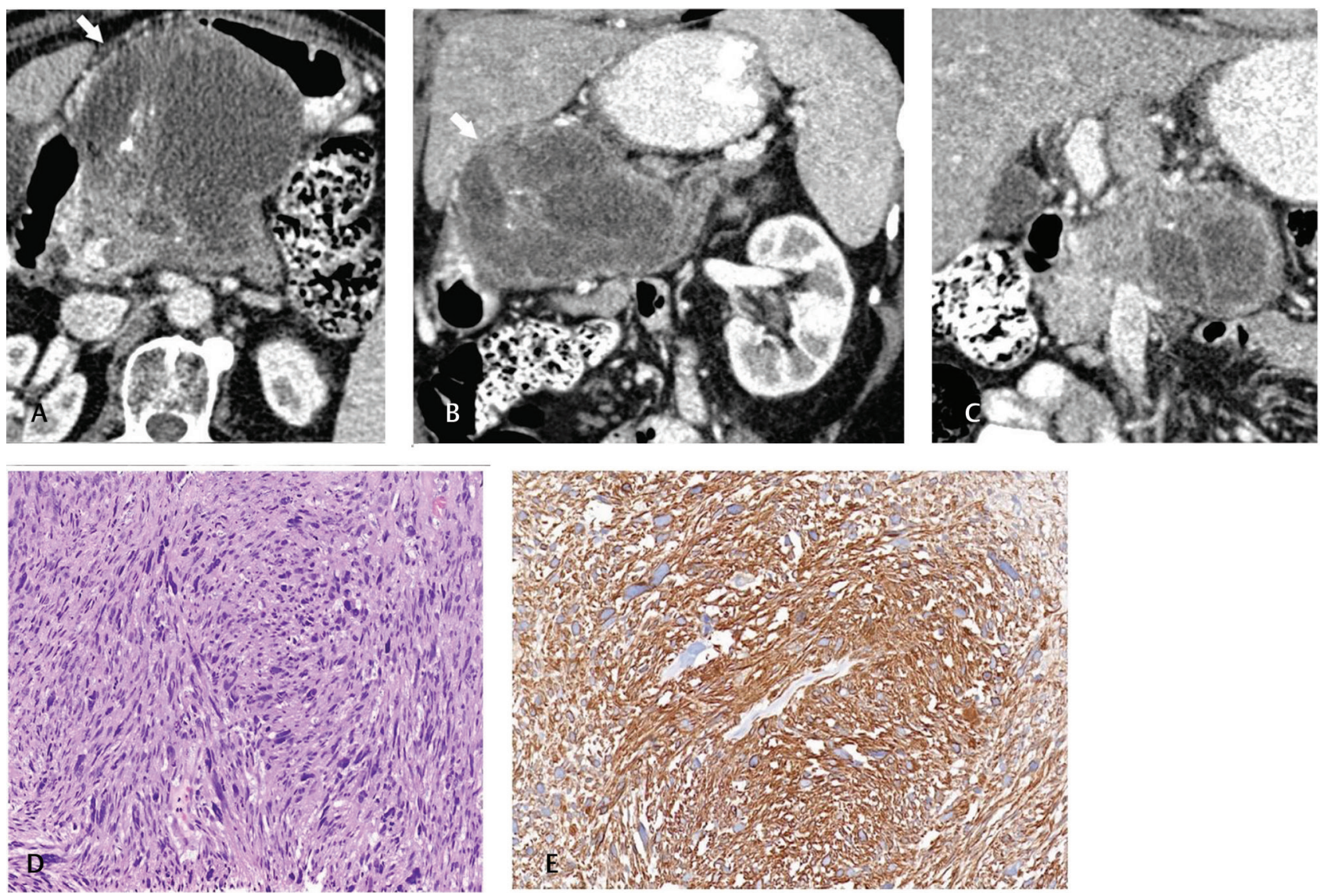

Fig. 6 (A-C) A 66-year-old man with pain in the abdomen. Axial and oblique CECT abdomen images show a heterogeneously enhancing mass in the body of the pancreas (arrows) with mild dilatation of the main pancreatic duct. The mass was biopsied with the pathology demonstrating leiomyosarcoma. (D, E) Hematoxylin and eosin stained section (10x) showing a high-grade pleomorphic sarcoma (D). Cells are arranged in fascicles. Marked nuclear pleomorphism and brisk mitotic activity are evident. On immunohistochemistry (E), the tumor cells show strong cytoplasmic positivity for antibody against smooth muscle actin (SMA), leading to a diagnosis of leiomyosarcoma.

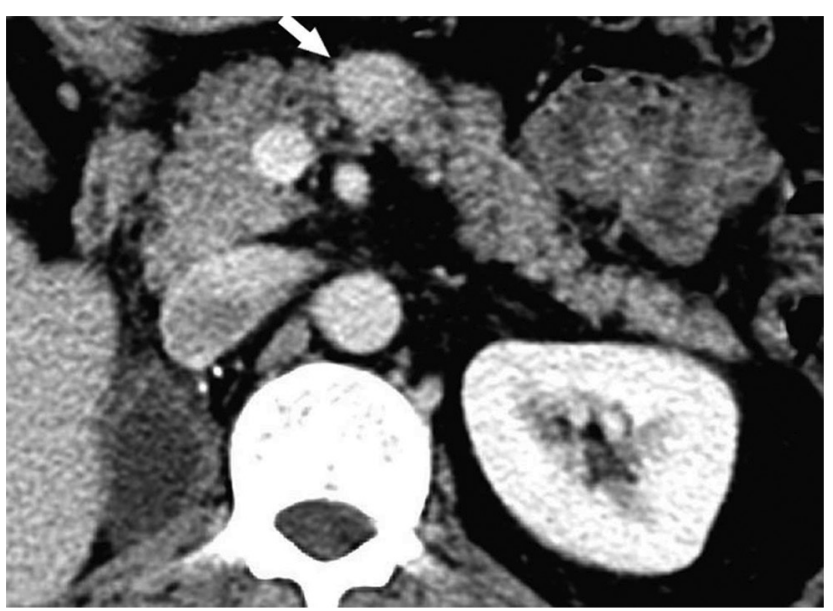

Fig. 7 A 49-year-old man, with history of renal cell carcinoma which was operated in 2012. A surveillance CECT performed in 2017 revealed a new enhancing nodule on the pancreatic body, consistent with metastasis.

study. Nonspecific symptoms like abdominal pain, gastrointestinal bleeding, and weight loss can be present. Occasionally, a pancreatic head metastasis can cause biliary obstruction and result in jaundice. Pancreatic ductal obstruction can lead to upstream parenchymal atrophy. ${ }^{33,34}$ Usually patients with pancreatic metastases would have concurrent widespread disease. In patients with isolated pancreatic involvement, surgical resection may offer survival benefit, especially when the metastasis is detected after a prolonged disease-free interval, suggestive of biological pattern of slow growth. ${ }^{34-39}$

On cross-sectional imaging, there are usually three patterns of pancreatic involvement-solitary mass, multifocal lesions, and a diffusely infiltrative lesion. ${ }^{40}$ Hyperenhancing pancreatic metastases include RCC and melanoma metastases, while lung, colonic, and gastric secondaries are usually hypoenhancing on contrast enhanced CT/MRI ( - Fig. 8). A commonly encountered feature among hypervascular metastases is that smaller tumors enhance homogenously and larger $(>1.5 \mathrm{~cm})$ lesions show peripheral enhancement due to central hypoperfusion/necrosis. ${ }^{34,41}$ Hypervascular metastases may show intense early enhancement on arterial phase, followed by washout on portal and delayed phase images. ${ }^{42,43}$

Differentials of hypervascular metastases include pancreatic neuroendocrine tumor and intrapancreatic accessory spleen. A relative percentage washout value $\left(R P W=\left(A_{a}-A_{p}\right)\right.$ $\times 100 / A_{a}$, where $A_{a}$ and $A_{p}$ are attenuation values of the pancreatic mass on 25 second arterial and 70 second portal $c$ ontrast-material-enhanced CT scan) of $19 \%\left(\mathrm{RCC}_{\text {median }}: 27.1\right.$; $\mathrm{NET}_{\text {median }}$ : 3.9) can be used to differentiate between RCC 
metastasis to the pancreas from pancreatic neuroendocrine tumor, in post nephrectomy surveillance patients. ${ }^{44}$ Accessory intrapancreatic spleen has attenuation similar to the spleen on contrast enhanced as well as unenhanced studies, including all MRI sequences. Definitive diagnosis can be established with scintigraphy using either technetium Tc 99m-labeled damaged red blood cell or technetium Tc 99m-sulfur colloid. Alternatively, the diagnosis can also be

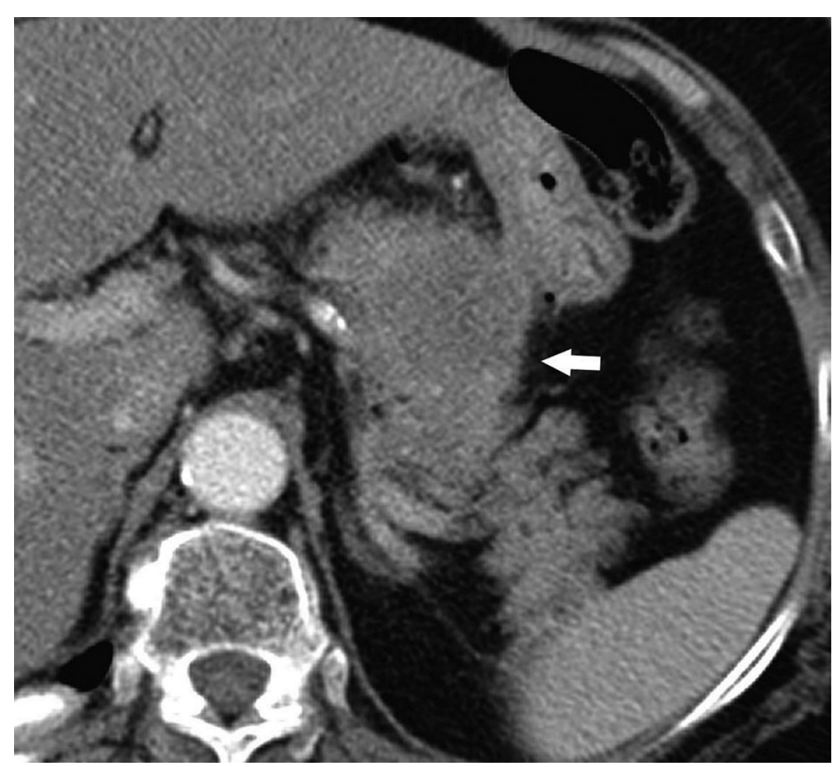

Fig. 8 A 67-year-old man with lung cancer. Axial CECT image shows a hypoenhancing lesion in the body of the pancreas, consistent with metastasis. confirmed on MRI using reticuloendothelial system specific contrast agent (superparamagnetic iron oxide)-enhanced study, where the lesion as well as spleen will show drop in signal intensity on T2 and T2* sequences. ${ }^{45}$ Solitary hypoenhancing metastasis can resemble pancreatic adenocarcinoma. A large pancreatic head mass without biliary/pancreatic ductal dilatation/posterior fat infiltration should raise a suspicion of nonpancreatic nature of the neoplasm. Metastases also tend to have features of the primary neoplasm. ${ }^{34}$

\section{Differential Diagnosis and Take-Home Points}

PDAC and neuroendocrine tumors are the most common solid pancreatic neoplasms, which need to be considered first in any case of a pancreatic mass. Any unusual features of presentation such as large size, heterogeneity with necrosis or hemorrhage, lack of ductal dilatation or jaundice, lack of parenchymal atrophy, or unusual age, should raise suspicion of a different etiology. ${ }^{3}$ Correlating the imaging findings with patient demographics, history, clinical symptoms, and laboratory parameters can help narrow the differentials. The pattern of enhancement, that is, hypoenhancing versus hypoenhancing/hypervascular lesions is often helpful. ${ }^{46}$ Hypoenhancing tumors resemble PDAC and include adenosquamous carcinoma, ACC, and nonepithelial neoplasms such as lymphoma and some metastases. Hyperenhancing masses can resemble pancreatic neuroendocrine tumors and include hypervascular metastases, and (rarely) solitary fibrous tumors. ${ }^{46}$ Having said that, image guided biopsy remains the mainstay for diagnosis, and attempting to give a definitive diagnosis based on imaging alone should be avoided in most cases ( - Table 2 ).

Table 2 Summary of clinicoradiological features of rare pancreatic neoplasms

\begin{tabular}{|c|c|c|c|c|c|}
\hline Tumor & Age & Gender & Clinical features & Location & Imaging findings \\
\hline $\begin{array}{l}\text { Pancreatic acinar cell } \\
\text { tumor }\end{array}$ & $\begin{array}{l}\text { 5th-7th } \\
\text { decade }\end{array}$ & $M \gg \gg>F$ & $\begin{array}{l}\text { Lipase hypersecretion syndrome } \\
\text { (subcutaneous fat necrosis, } \\
\text { polyarthritis) }\end{array}$ & $\begin{array}{l}\text { Any site in } \\
\text { the pancreas }\end{array}$ & $\begin{array}{l}\text { Hypoenhancing similar to PDAC } \\
\text { with frequent cystic/necrotic } \\
\text { component and calcifications }\end{array}$ \\
\hline Pancreatoblastoma & 1st decade & $M>>F$ & $\begin{array}{l}\text { Large abdominal mass with } \\
\text { pressure symptoms }\end{array}$ & Any part & $\begin{array}{l}\text { Well-defined to partially circum- } \\
\text { scribed, lobulated mass } \\
\text { Can be cystic }\end{array}$ \\
\hline $\begin{array}{l}\text { Perivascular epithelioid } \\
\text { cell tumor }\end{array}$ & & $\mathrm{F} \gg \gg \gg$ & $\begin{array}{l}\text { Frequently associated with } \\
\text { tuberous sclerosis }\end{array}$ & & $\begin{array}{l}\text { Well-defined, encapsulated, and } \\
\text { hypoenhancing pancreatic mass }\end{array}$ \\
\hline $\begin{array}{l}\text { Adenosquamous } \\
\text { carcinoma }\end{array}$ & 7th decade & $\mathrm{M}>\mathrm{F}$ & $\begin{array}{l}\text { Weight loss, anorexia, jaundice, } \\
\text { abdominal and back pain are } \\
\text { similar to PDAC }\end{array}$ & Head & $\begin{array}{l}\text { Round-lobulated lesions associat- } \\
\text { ed with extensive necrosis } \\
\text { Tumor thrombus in portal vein }\end{array}$ \\
\hline $\begin{array}{l}\text { Solitary fibrous tumor } \\
\text { (SFT) }\end{array}$ & $\begin{array}{l}\text { 5th-8th } \\
\text { decade }\end{array}$ & $\mathrm{F} \gg>M$ & $\begin{array}{l}\text { Abdominal distension, bowel } \\
\text { obstruction, or constipation } \\
\text { Hypoglycemia (Doege-Potter } \\
\text { syndrome) } \\
\text { Clubbing, hypertrophic osteoar- } \\
\text { thropathy, and arthralgia }\end{array}$ & Head & $\begin{array}{l}\text { Large hypervascular mass with } \\
\text { persistent progressive enhance- } \\
\text { ment on multiphase studies }\end{array}$ \\
\hline Lymphoma & 7th decade & $M>>F$ & $\begin{array}{l}\text { Asymptomatic or abdominal } \\
\text { pain } \\
\text { B symptoms like fever, night } \\
\text { sweats, and weight loss }\end{array}$ & $\begin{array}{l}\text { Head } \\
\text { >>body/tail }\end{array}$ & $\begin{array}{l}\text { Focal lesion } \\
\text { or } \\
\text { diffuse, ill-defined, infiltrative } \\
\text { mass } \\
\text { No ductal dilatation and vascular } \\
\text { occlusion/invasion, even if large } \\
\text { Associated large adenopathy }\end{array}$ \\
\hline Sarcomas & $\begin{array}{l}\text { Usually } \\
\text { older age } \\
\text { group }\end{array}$ & & Abdominal distention or pain & Any & $\begin{array}{l}\text { Large, heterogeneous, masses } \\
\text { without a distinguishing imaging } \\
\text { feature }\end{array}$ \\
\hline
\end{tabular}

Abbreviations: M, male; F, female; PDAC, pancreatic ductal adenocarcinoma. 


\section{Conflict of Interest}

None declared.

\section{Acknowledgment}

The authors wish to acknowledge Dr. Atul Shinagare, Assistant Professor, Department of Radiology, Dana Farber Cancer Institute, Harvard Medical School, for contributing one of the figures for the manuscript.

\section{References}

1 Shah S, Mortele KJ. Uncommon solid pancreatic neoplasms: ultrasound, computed tomography, and magnetic resonance imaging features. Semin Ultrasound CT MR 2007;28(5):357-370

2 Manning MA, Paal EE, Srivastava A, Mortele KJ. Nonepithelial neoplasms of the pancreas, part 2: malignant tumors and tumors of uncertain malignant potential. Radiographics 2018;38(4):1047-1072

3 Manning MA, Srivastava A, Paal EE, Gould CF, Mortele KJ. Nonepithelial neoplasms of the pancreas: radiologic-pathologic correlation, part 1-benign tumors. Radiographics 2016;36(1):123-141

4 Radin DR, Colletti PM, Forrester DM, Tang WW. Pancreatic acinar cell carcinoma with subcutaneous and intraosseous fat necrosis. Radiology 1986;158(1):67-68

5 Tatli S, Mortele KJ, Levy AD, et al. CT and MRI features of pure acinar cell carcinoma of the pancreas in adults. Am J Roentgenol 2005;184(2):511-519

6 Raman SP, Hruban RH, Cameron JL, Wolfgang CL, Kawamoto S, Fishman EK. Acinar cell carcinoma of the pancreas: computed tomography features-a study of 15 patients. Abdom Imaging 2013;38(1):137-143

7 Bhosale P, Balachandran A, Wang H, et al. CT imaging features of acinar cell carcinoma and its hepatic metastases. Abdom Imaging 2013;38(6):1383-1390

8 Raman SP, Hruban RH, Cameron JL, Wolfgang CL, Fishman EK. Pancreatic imaging mimics: part 2, pancreatic neuroendocrine tumors and their mimics. Am J Roentgenol 2012;199(2):309-318

9 Kitagami H, Kondo S, Hirano S, Kawakami H, Egawa S, Tanaka M. Acinar cell carcinoma of the pancreas: clinical analysis of 115 patients from Pancreatic Cancer Registry of Japan Pancreas Society. Pancreas 2007;35(1):42-46

10 Boyd CA, Benarroch-Gampel J, Sheffield KM, Cooksley CD, Riall TS. 415 patients with adenosquamous carcinoma of the pancreas: a population-based analysis of prognosis and survival. J Surg Res 2012;174(1):12-19

11 Toshima F, Inoue D, Yoshida K, et al. Adenosquamous carcinoma of pancreas: CT and MR imaging features in eight patients, with pathologic correlations and comparison with adenocarcinoma of pancreas. Abdom Radiol (NY) 2016;41(3):508-520

12 Low G, Panu A, Millo N, Leen E. Multimodality imaging of neoplastic and nonneoplastic solid lesions of the pancreas. Radiographics 2011;31(4):993-1015

13 Shet NS, Cole BL, Iyer RS. Imaging of pediatric pancreatic neoplasms with radiologic-histopathologic correlation. Am J Roentgenol 2014;202(6):1337-1348

14 Boninsegna E, Zamboni GA, Facchinelli D, et al. CT imaging of primary pancreatic lymphoma: experience from three referral centres for pancreatic diseases. Insights Imaging 2018;9(1):17-24

15 Dawson IMP, Cornes JS, Morson BC. Primary malignant lymphoid tumours of the intestinal tract. Report of 37 cases with a study of factors influencing prognosis. Br J Surg $1961 ; 49(213): 80-89$
16 Anand D, Lall C, Bhosale P, Ganeshan D, Qayyum A. Current update on primary pancreatic lymphoma. Abdom Radiol (NY) 2016;41(2):347-355

17 Sadot E, Yahalom J, Do RKG, et al. Clinical features and outcome of primary pancreatic lymphoma. Ann Surg Oncol 2015;22(4):1176-1184

18 Sahani DV, Bonaffini PA, Catalano OA, Guimaraes AR, Blake MA. State-of-the-art PET/CT of the pancreas: current role and emerging indications. Radiographics 2012;32(4):1133-1158, discussion 1158-1160

19 Hall MN, Jagannathan JP, Ramaiya NH, Shinagare AB, Van den Abbeele AD. Imaging of extraosseous myeloma: CT, PET/CT, and MRI features. Am J Roentgenol 2010;195(5):1057-1065

20 Baez JC, Landry JM, Saltzman JR, Qian X, Zinner MJ, Mortelé KJ. Pancreatic PEComa (sugar tumor): MDCT and EUS features. JOP 2009;10(6):679-682

21 Tirumani SH, Shinagare AB, Hargreaves J, et al. Imaging features of primary and metastatic malignant perivascular epithelioid cell tumors. Am J Roentgenol 2014;202(2):252-258

22 Hornick JL, Fletcher CDM. PEComa: what do we know so far.? Histopathology 2006;48(1):75-82

23 Shanbhogue AK, Prasad SR, Takahashi N, Vikram R, Zaheer A, Sandrasegaran K. Somatic and visceral solitary fibrous tumors in the abdomen and pelvis: cross-sectional imaging spectrum. Radiographics 2011;31(2):393-408

24 Ginat DT, Bokhari A, Bhatt S, Dogra V. Imaging features of solitary fibrous tumors. Am J Roentgenol 2011;196(3):487-495

25 Delgado J, Jaramillo D, Ho-Fung V, Fisher MJ, Anupindi SA. MRI features of plexiform neurofibromas involving the liver and pancreas in children with neurofibromatosis type 1 . Clin Radiol 2014;69(6):e280-e284

26 Lee NJ, Hruban RH, Fishman EK. Abdominal schwannomas: review of imaging findings and pathology. Abdom Radiol (NY) 2017;42(7):1864-1870

27 Gupta A, Subhas G, Mittal VK, Jacobs MJ. Pancreatic schwannoma: literature review. J Surg Educ 2009;66(3):168-173

28 Novellas S, Chevallier P, Saint Paul MC, Gugenheim J, Bruneton JN. MRI features of a pancreatic schwannoma. Clin Imaging 2005;29(6):434-436

29 England RJ, Woodley H, Cullinane C, McClean P, Walker J, Stringer MD. Pediatric pancreatic hemangioma: a case report and literature review. JOP 2006;7(5):496-501

30 Kobayashi H, Itoh T, Murata R, Tanabe M. Pancreatic cavernous hemangioma: CT, MRI, US, and angiography characteristics. Gastrointest Radiol 1991;16(4):307-310

31 Mundinger GS, Gust S, Micchelli ST, Fishman EK, Hruban RH, Wolfgang CL. Adult pancreatic hemangioma: case report and literature review. Gastroenterol Res Pract 2009;2009:839730

32 Mao Y, Sang X, Liang N, et al. Peripheral primitive neuroectodermal tumors arising in the pancreas: the first case report in Asia and a review of the 14 total reported cases in the world. Hepatobiliary Surg Nutr 2013;2(1):51-60

33 Klein KA, Stephens DH, Welch TJ. CT characteristics of metastatic disease of the pancreas. Radiographics 1998;18(2):369-378

34 Triantopoulou C, Kolliakou E, Karoumpalis I, Yarmenitis S, Dervenis C. Metastatic disease to the pancreas: an imaging challenge. Insights Imaging 2012;3(2):165-172

35 Tanis PJ, van der Gaag NA, Busch ORC, van Gulik TM, Gouma DJ. Systematic review of pancreatic surgery for metastatic renal cell carcinoma. Br J Surg 2009;96(6):579-592

36 Z'graggen K, Fernández-del Castillo C, Rattner DW, Sigala H, Warshaw AL. Metastases to the pancreas and their surgical extirpation. Arch Surg 1998;133(4):413-417, discussion 418-419

37 Zerbi A, Ortolano E, Balzano G, Borri A, Beneduce AA, Di Carlo V. Pancreatic metastasis from renal cell carcinoma: which 
patients benefit from surgical resection? Ann Surg Oncol 2008;15(4):1161-1168

38 Reddy S, Wolfgang CL. The role of surgery in the management of isolated metastases to the pancreas. Lancet Oncol 2009;10(3):287-293

39 Konstantinidis IT, Dursun A, Zheng $\mathrm{H}$, et al. Metastatic tumors in the pancreas in the modern era. J Am Coll Surg 2010;211(6):749-753

40 Merkle EM, Boaz T, Kolokythas O, Haaga JR, Lewin JS, Brambs HJ. Metastases to the pancreas. $\mathrm{Br} \mathrm{J}$ Radiol 1998;71(851):1208-1214

41 Tan CH, Tamm EP, Marcal L, et al. Imaging features of hematogenous metastases to the pancreas: pictorial essay. Cancer Imaging 2011;11(1):9-15

42 Ascenti G, Visalli C, Genitori A, Certo A, Pitrone A, Mazziotti S. Multiple hypervascular pancreatic metastases from renal cell carcinoma: dynamic MR and spiral CT in three cases. Clin Imaging 2004;28(5):349-352

43 Ahmed S, Johnson PT, Hruban R, Fishman EK. Metastatic disease to the pancreas: pathologic spectrum and CT patterns. Abdom Imaging 2013;38(1):144-153

44 Kang TW, Kim SH, Lee J, et al. Differentiation between pancreatic metastases from renal cell carcinoma and hypervascular neuroendocrine tumour: use of relative percentage washout value and its clinical implication. Eur J Radiol 2015;84(11):2089-2096

$45 \mathrm{Kim} \mathrm{SH}$, Lee JM, Han JK, et al. Intrapancreatic accessory spleen: findings on MR Imaging, CT, US and scintigraphy, and the pathologic analysis. Korean J Radiol 2008;9(2):162-174

46 Steinman J, Zaheer A, Kluger MD, Remotti H, Hecht EM. Rare pancreatic tumors. Abdom Radiol (NY) 2018;43(2):285-300 